EPJ Web of Conferences 108, 02004 (2016)

DOI: $10.1051 /$ epjconf/201610802004

(C) Owned by the authors, published by EDP Sciences, 2016

\title{
Multi-Loop Calculations of Anomalous Exponents in the Models of Critical Dynamics
}

\author{
L. Ts. Adzhemyan ${ }^{1}$, M. Dančo ${ }^{2,3, a}$, M. Hnatič ${ }^{2,3,4, b}$, E. V. Ivanova ${ }^{1}$, and M. V. Kompaniets ${ }^{1}$ \\ ${ }^{1}$ Department of Theoretical Physics, St. Petersburg State University, Uljanovskaja 1, 198504 St. Petersburg, \\ Petrodvorez, Russia \\ ${ }^{2}$ Bogoliubov Laboratory of Theoretical Physics, Joint Institute for Nuclear Research, Joliot-Curie 6, 141980 \\ Dubna, Moscow Region, Russia \\ ${ }^{3}$ Department of Theoretical Physics, SAS, Institute of Experimental Physics, Watsonova 47, 04001 Košice, \\ Slovakia \\ ${ }^{4}$ Faculty of Science, P. J. Šafárik University, Šrobárova 2, 04154 Košice
}

\begin{abstract}
The Renormalization group method (RG) is applied to the investigation of the $\mathrm{E}$ model of critical dynamics, which describes the transition from the normal to the

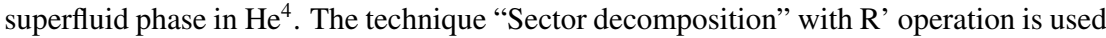
for the calculation of the Feynman diagrams. The RG functions, critical exponents and critical dynamical exponent $z$, which determines the growth of the relaxation time near the critical point, have been calculated in the two-loop approximation in the framework of $\varepsilon$-expansion. The relevance of a fixed point for helium, where the dynamic scaling is weakly violated, is briefly discussed.
\end{abstract}

\section{Introduction}

The renormalization group method (RG) for the study of phase transitions and critical phenomena [1-3] allows one to justify the critical scaling and gives a recipe for calculating critical exponents as expansions in a small parameter $\varepsilon=\left(d_{c}-d\right) / 2$, which is the deviation from the critical dimension $d_{c}=4$. The calculation of the renormalization-group functions is the main technical problem. Usually, it is solved by determining the renormalization constants from calculations of the corresponding Feynman diagrams. In this case, the analytical calculation of loop diagrams in higher order is quite complicated, so it is convenient to use numerical methods that allow subsequently to automate the process of search of the renormalization-group functions. The complexity of the numerical calculations resides in the presence of the $\varepsilon$-poles in the renormalization constants. There are several ways to solve the problem. In this paper, we will use the sector decomposition [4] which isolates the divergences from the parameter integrals occurring in the perturbative quantum field theory.

\footnotetext{
a e-mail: danco@saske.sk

be-mail: hnatic@saske.sk
}

This is an Open Access article distributed under the terms of the Creative Commons Attribution License 4.0, which permits unrestricted use, distribution, and reproduction in any medium, provided the original work is properly cited. 


\section{Formulation of the problem}

The standard Martin-Siggia-Rose mechanism [5] leads to a field theory action of the model E in the form

$$
\begin{aligned}
S & =2 \lambda_{0} \psi^{+\prime} \psi^{\prime}-\lambda_{0} u_{0} m^{\prime} \partial^{2} m^{\prime} \\
& +\psi^{+}\left\{-\partial_{t} \psi+\lambda_{0}\left[\partial^{2} \psi-\tau_{0} \psi-g_{10}\left(\psi^{+} \psi\right) \psi / 3\right]+i \lambda_{0} g_{30} \psi[-m+h]\right\} \\
& +\psi^{\prime}\left\{-\partial_{t} \psi^{+}+\lambda_{0}\left[\partial^{2} \psi^{+}-\tau_{0} \psi^{+}-g_{10}\left(\psi^{+} \psi\right) \psi^{+} / 3\right]-i \lambda_{0} g_{30} \psi^{+}[-m+h]\right\} \\
& +m^{\prime}\left\{-\partial_{t} m-\lambda_{0} u_{0} \partial^{2}[-m+h]+i \lambda_{0} g_{30}\left[\psi^{+} \partial^{2} \psi-\psi \partial^{2} \psi^{+}\right]\right\}
\end{aligned}
$$

with the real one-component field $m$ and the complex $N$-component fields $\psi$ and $\psi^{+}$. The real problem for superfluid $\mathrm{He}^{4}$ corresponds to $\mathrm{N}=1$ ( $n=2$ in terms of the $\mathrm{O}(\mathrm{n})$ group of symmetry). The action (1) is unrenormalized with the corresponding bare parameters $\lambda_{0}, u_{0}, \tau_{0}$ and coupling constants $g_{10}, g_{30}$. All possible counterterms of the dynamical model have the form of the terms in (1), and hence they are reproduced by the introduction of the needed renormalization constants. The model is multiplicatively renormalizable because the unrenormalized action is related to the renormalized one by the standard procedure of multiplicative renormalization $S_{R}(\varphi, e)=S\left(Z_{\varphi} \varphi, e_{0}\right)$, where the functional variable

$$
Z_{\varphi} \varphi \equiv\left\{Z_{\psi} \psi, Z_{\psi^{\prime}} \psi^{\prime}, Z_{\psi^{+}} \psi^{+}, Z_{\psi^{+}} \psi^{+^{\prime}}, Z_{m} m, Z_{m^{\prime}} m^{\prime}\right\}
$$

denotes the field renormalization. The bare parameters and coupling constants $e_{0} \equiv \lambda_{0}, u_{0}, \tau_{0}, g_{10}, g_{30}$ are connected with their renormalized partners $e \equiv \lambda, u, \tau, g_{1}, g_{3}$ by the expressions

$$
\lambda_{0}=\lambda Z_{\lambda}, \quad u_{0}=u Z_{u}, \quad \tau_{0}=\tau Z_{\tau}, \quad g_{10}=g_{1} \mu^{2 \varepsilon} Z_{g_{1}}, \quad g_{30}=g_{3} \mu^{\varepsilon} Z_{g_{3}},
$$

where $Z$ with corresponding subscripts indicate the renormalization constants. The model is logarithmic for $\varepsilon=0$ (bare coupling constants become dimensionless). In this case, the Feynman graphs which are necessary for the calculation of the renormalization constants $Z$ contain ultraviolet (UV) divergences in all orders of perturbation theory. We use the dimensional regularization and minimal substraction (MS) scheme, where the divergent parts of the graphs manifest themselves as poles in $\varepsilon$. Due to the absence of some divergences, the following relation must be satisfied:

$$
Z_{m^{\prime}} Z_{m}=1, \quad Z_{\lambda} Z_{g_{3}}=Z_{m} .
$$

\section{The method of sector decompostion}

The algorithm for searching the divergent part (singular term) of the individual graphs involves three steps. In the first, the integration region is divided into sectors, each of which contains isolated singularities. In the second step, the coefficients at all poles in $\varepsilon$ are extracted. In the last step, Feynman parameters are integrated analytically or numerically if an analytical treatment is not accessible.

For comparison with De Dominicis, Peliti $[6,7]$ we divided our diagrams into groups. Here we will show how to calculate one of the groups using the sector decomposition procedure

$$
-\frac{g_{3}^{4}}{(2 \pi)^{d}} \int d^{d} \vec{k} \int d^{d} \vec{q} \frac{1}{\left(k^{2} u+k^{2}+\tau\right)\left(q^{2} u+q^{2}+\tau\right)\left((k-q)^{2}+\tau\right)\left(u\left(k^{2}+q^{2}\right)+(k-q)^{2}+\tau\right)} .
$$

Using the Feynman parametrization

$$
A_{1}^{-\lambda_{1}} \ldots A^{-\lambda_{l}}=\frac{\Gamma\left(\sum_{i=1}^{l} \lambda_{i}\right)}{\prod_{i=1}^{l} \Gamma\left(\lambda_{i}\right)} \int_{0}^{1} \ldots \int_{0}^{1} d v_{1} \ldots d v_{l} \frac{\delta\left(\sum_{i=1}^{l} v_{i}-1\right) \Pi_{i=1}^{l} v_{i}^{\lambda_{i}-1}}{\left[\sum_{i=1}^{l} A_{i} v_{i}\right]^{\sum_{i=1}^{l} \lambda_{i}}}
$$


and the general $l$-loop integral with an arbitrary power of the quadratic form [2]

$$
\frac{1}{(2 \pi)^{n d}} \int \ldots \int \frac{d^{d} \vec{k}_{1} \ldots d^{d} \vec{k}_{n}}{\left[V_{i s}\left(\mathbf{k}_{i}, \mathbf{k}_{s}\right)+2\left(\mathbf{a}_{i}, \mathbf{k}_{i}\right)+c\right]^{\alpha}}=\frac{(4 \pi)^{-n d / 2} \Gamma\left(\alpha-\frac{n d}{2}\right)(\operatorname{det} V)^{-d / 2}}{\Gamma(\alpha)\left[c-\left(V^{-1}\right)_{i s}\left(\mathbf{a}_{i}, \mathbf{a}_{s}\right)\right]^{\alpha-n d / 2}}
$$

we obtain the integral (5) as

$$
-g_{3}^{4} \frac{\Gamma(2 \varepsilon) \Gamma^{2}(2-\varepsilon)}{4} \int_{0}^{1} d v_{1} \ldots \int_{0}^{1} d v_{4} \frac{\delta\left(1-v_{1}-v_{2}-v_{3}-v_{4}\right)}{(\operatorname{det} V)^{2-\varepsilon}},
$$

where the determinant is

$$
\operatorname{det} V=(1+u)^{2}\left(v_{2} v_{3}+v_{2} v_{4}+v_{3} v_{4}\right)+(1+u)\left(v_{1} v_{2}+v_{1} v_{3}\right)+2 u v_{1} v_{4}+u(2+u) v_{4}^{2} .
$$

The idea of the sector decomposition is essential for the first step of the algorithm. It is based on splitting the integration region to achieve a disentanglement of the singularities. The integral in (8) can be split into the sectors

$$
\begin{aligned}
I & =I_{1(2,3,4) 4(2,3)}+I_{1(2,3,4) 3(2,4)}+I_{1(2,3,4) 2(3,4)}+I_{2(1,3,4) 1(3,4)}+I_{2(1,3,4) 3(1,4)}+I_{2(1,3,4) 4(1,3)} \\
& +I_{3(1,2,4) 1(2,4)}+I_{3(1,2,4) 2(1,4)}+I_{3(1,2,4) 4(1,2)}+I_{4(1,2,3)},
\end{aligned}
$$

where, for instance, $I_{4(1,2,3)}$ means that the integration domain is split into the part where $v_{4}$ is always larger than $v_{1}, v_{2}, v_{3}$. The splitting can be translated into a change of the integration boundaries using the transformation

$$
v_{4} \rightarrow v_{4}, \quad v_{1} \rightarrow v_{4} \tilde{v}_{1}, \quad v_{2} \rightarrow v_{4} \tilde{v}_{2}, \quad v_{3} \rightarrow v_{4} \tilde{v}_{3}
$$

and then the integral is remapped onto the unit hypercube. Therefore, the sector $I_{4(1,2,3)}$ after the change of the integration boundaries looks like

$$
\begin{aligned}
& \int_{0}^{1} d v_{1} \int_{0}^{1} d v_{2} \int_{0}^{1} d v_{3} \frac{1}{\left[(1+u)^{2}\left(v_{2} v_{3}+v_{2}+v_{3}\right)+(1+u)\left(v_{1} v_{2}+v_{1} v_{3}\right)+2 u v_{1}+u(2+u)\right]^{2-\varepsilon}} \\
& =\frac{5 \log (5)+35 \log (7)-22 \log (11)-21 \log (3)}{2 u(1+u)^{2}(1+2 u)} .
\end{aligned}
$$

The final result for the integral (8) reads

$$
-g_{3}^{4} \frac{\Gamma[2 \varepsilon] \Gamma^{2}[2-\varepsilon]}{2^{2}} I=\frac{g_{3}^{4}}{4 u(1+u)^{2}(2 \varepsilon)}[u \log (u)-2(1+u) \log (1+u)+(2+u) \log (2+u)-\log (4)]
$$

which is exactly that was obtained by De Dominicis and Peliti for this group [7].

\section{RG functions and scaling regimes}

The RG analysis leads to the conclusion that the possible scaling regimes are given by the IR stable fixed points of the corresponding RG equations. The fixed points of the RG equations can be found from the requirement that all the beta functions of the model vanish and the IR stability of the fixed point is given by the requirement that all the eigenvalues $\omega$ of the matrix of the first derivatives $\omega_{i k}=\partial \beta_{i} / \partial g_{k}$ at the fixed point must have positive real parts, where $\beta_{i}$ is the full set of $\beta$-functions and $g_{k}$ is the full set of charges $\left\{g_{1}, g_{3}, u\right\}$. Explicit calculations show that the variables $f$ and $w$ defined as follows:

$$
f=\frac{g_{3}^{2}}{8 \pi^{2} u}, \quad w=\frac{1}{u}
$$


are more convenient for the RG analysis. For the dynamical $\beta$-functions of the model $\mathrm{E}$ we have

$$
\beta_{f}=f\left[-2 \varepsilon-\gamma_{w}+2 \gamma_{\lambda}\right], \quad \beta_{w}=-w \gamma_{w} .
$$

From the knowledge of the renormalization constants $\mathrm{Z}$ we obtain two loop results for $\gamma$ functions in the form

$$
\begin{aligned}
& \gamma_{\lambda}=\frac{f}{1+w}-f^{2} F(w)-\frac{g_{1}^{2}}{18}\left[6 \ln \left(\frac{4}{3}\right)-1\right] \\
& \gamma_{w}=f\left[\frac{1}{1+w}-\frac{1}{2}\right]+f^{2}[H(w)-F(w)]-\frac{g_{1}^{2}}{18}\left[6 \ln \left(\frac{4}{3}\right)-1\right],
\end{aligned}
$$

where

$$
\begin{aligned}
& H(w)=\frac{1}{4(1+w)}\left[w^{2}(2+w) \ln \left(\frac{(1+w)^{2}}{w(2+w)}\right)-w-\frac{1}{2}\right], \\
& F(w)=\frac{1}{8(1+w)^{3}}\left[4(1+2 w) \ln \left(\frac{(1+w)^{2}}{(1+2 w)}\right)+54(1+w) \ln \left(\frac{4}{3}\right)-8 w-12\right] .
\end{aligned}
$$

The fixed point values $f^{*}$ and $w^{*}$ are determined by the zeros of the $\beta$-functions (15). For the dynamical fixed point with

$$
f^{*}=(2 \varepsilon)+\frac{3}{8}\left[2 \ln \left(\frac{4}{3}\right)-1\right](2 \varepsilon)^{2}, \quad w^{*}=1+\frac{1}{50}\left[29-324 \ln \left(\frac{4}{3}\right)\right](2 \varepsilon)
$$

we have $\gamma_{w}^{*}=0, \gamma_{\lambda}^{*}=\varepsilon$, and hence for the exponent $z$ we obtain the exact expression

$$
z=2-\gamma_{\lambda}^{*}=2-\varepsilon=\frac{d}{2} .
$$

There exists another fixed point

$$
w^{*}=0 \quad f^{*}=\frac{2}{3}(2 \varepsilon)+\frac{2}{675}\left[702 \ln \left(\frac{4}{3}\right)-167\right](2 \varepsilon)^{2},
$$

where the RG functions are in the form

$$
\gamma_{\lambda}^{*}=\varepsilon+\frac{\gamma_{w}^{*}}{2}, \quad \gamma_{w}^{*}=\frac{(2 \varepsilon)}{3}+\left[\frac{259}{675}-\frac{52}{25} \ln \left(\frac{4}{3}\right)\right](2 \varepsilon)^{2}
$$

and then in this case the exponent $z$ is nontrivial

$$
z=2-\gamma_{\lambda}^{*}=\frac{d}{2}-\frac{\gamma_{w}^{*}}{2}
$$

For $d=4-2 \varepsilon$ the exponents $\omega$ associated with the charges $f$ and $w$ are

$$
\begin{aligned}
& \omega_{f}=(2 \varepsilon)+\left[\frac{103}{200}-\frac{259}{100} \ln \left(\frac{4}{3}\right)\right](2 \varepsilon)^{2}=(2 \varepsilon)-0.230097(2 \varepsilon)^{2} \\
& \omega_{w}=\frac{(2 \varepsilon)}{4}+\left[\frac{347}{800}-\frac{1507}{800} \ln \left(\frac{4}{3}\right)\right](2 \varepsilon)^{2}=\frac{(2 \varepsilon)}{4}-0.108171(2 \varepsilon)^{2}
\end{aligned}
$$


for the point (20) and

$$
\begin{aligned}
& \omega_{f}=(2 \varepsilon)+\left[\frac{158}{225}-\frac{72}{25} \ln \left(\frac{4}{3}\right)\right](2 \varepsilon)^{2}=(2 \varepsilon)-0.126302(2 \varepsilon)^{2} \\
& \omega_{w}=-\frac{(2 \varepsilon)}{3}+\left[\frac{52}{25} \ln \left(\frac{4}{3}\right)-\frac{259}{675}\right](2 \varepsilon)^{2}=-\frac{(2 \varepsilon)}{3}+0.214675(2 \varepsilon)^{2}
\end{aligned}
$$

for the point (22). The last one is called a weak scaling point and its possible relevance was discovered by De Dominicis and Peliti $[6,7]$. However, the authors made a numerical error in the evaluation of the corresponding diagrams, which was pointed out by Dohm [8]. Our results coincide with those obtained by Dohm [8]. As stated by all the aforementioned authors and A.N.Vasiliev [2], at small $\varepsilon$ the dynamical regime (21) is IR-stable for $n=2$. However, owing to the numerical smallness of the coefficients of the $\varepsilon$-expansions of $\omega_{w}$ we cannot discard the possibility that in the real dimension $d=3(2 \varepsilon=1)$ the sign of the exact $\omega_{w}$ is different from that obtained in lower orders in $\varepsilon$; hence the weak scaling regime (24) can still be IR stable for $n=2, d=3$. This means that the two loop calculations do not lead to the decision which of the fixed points is true because of the lack of $\omega$ calculation accuracy. The next order of the perturbation theory can change the sign of $\omega$ and modify the stability conditions.

\section{Conclusion}

We have studied the model $\mathrm{E}$ and calculated the RG functions up to the second order of the perturbation theory using the technique called the sector decomposition and partly discussed the relevance of a fixed point, where dynamic scaling is weakly violated. The physical interest is the recovery of the dynamical critical exponent $z$ which determines the growth of the relaxation time near the critical point. Multi-charged models can have different fixed points which correspond to different values of $z$. The first question that has to be solved is which of these points is stable and corresponds to the physical reality. The available results do not allow us to give a clear answer to this question; therefore, an urgent task is to calculate higher order terms of the perturbation theory.

\section{Acknowledgements}

This work was supported by Vega grant 1/0222/13 and 2/0045/13. L.Ts.A., M.V.K. and E.V.I. acknowledge the Saint Petersburg State University for the research grant 11.38.185.2014. We would like also to thank dr. Martin Val'a the coordinator of the project "Slovak infrastructure for high performance computing (SIVVP), ITMS 26230120002”.

\section{References}

[1] Hohenberg, P. C. and Halperin, B., I. Rev. Modern Phys. 49, 435-479 (1977)

[2] A. N. Vasiliev, The Field Theoretic Renormalization Group in Critical Behavior Theory and Stochastic Dynamics, Chapman and Hall/CRC, Boca Raton, Fla. (2004)

[3] Folk, R. and Moser, G., J. Phys. A: Math. Gen. 39, R207 (2006)

[4] Binoth, T., Heinrich, G., Nucl. Phys. B585 741-759 (2000)

[5] Martin, P. C., Siggia, E. D., and Rose, H. A., Phys. Rev. A 8, 423-437 (1973)

[6] De Dominicis, C. and Peliti, L., Phys. Rev. Lett. 38, 505 (1977)

[7] De Dominicis, C. and Peliti, L., Phys. Rev. B 18, 353 (1978)

[8] Dohm, V., Z. Physik B 33, 79-95 (1979) 
